\title{
Urethral ultrasonography: A novel diagnostic tool for dysuria following bipolar transurethral plasma kinetic prostatectomy
}

\author{
Guang-Chun Wang ${ }^{\mathrm{a}, 1}$, Cui-Dong Bian ${ }^{\mathrm{b}, 1}$, Ting-Ting Zhou ${ }^{\mathrm{a}}$, Min Liu ${ }^{\mathrm{a}}$, Jian-Hua Huang ${ }^{\mathrm{a}}$ and \\ Bo Peng ${ }^{\mathrm{a}, *}$ \\ ${ }^{a}$ Department of Urology, Shanghai Tenth People's Hospital, Tongji University School of Medicine, \\ Shanghai, China \\ ${ }^{\mathrm{b}}$ Department of Urology, Tongji Hospital, Tongji University School of Medicine, Shanghai, China
}

\begin{abstract}
Urethral ultrasonography is non-invasive and able to indicate the urethral lumen clearly, as well as the surrounding tissues of the posterior urethra, without contrast agent or X-ray irradiation. In this paper, we evaluate the reliability of urethral ultrasonography in the diagnosis of dysuria following bipolar transurethral plasma kinetic prostatectomy (TUPKP). A total of 120 benign prostate hyperplasia (BPH) patients with dysuria undergoing TUPKP were enrolled in this study, with a mean age of 72.8 years. All the patients received urethral ultrasonography, urethroscopy and bladder neck urethra stenosis oulectomy. Among the 120 cases, there were 22 cases of bladder neck closure, 20 bladder orifice stricture, 60 urethral stricture, 10 prostate remnants, 2 calculi in prostatic urethra, 4 dysfunction of bladder detrusor muscle and 2 flap of internal urethral orifice. $\chi^{2}$-test was used for the comparison of ultrasonography and urethral cystoscopy in the diagnosis of dysuria following TRPKP, and no significant difference was found between two diagnostic tools $\left(\chi^{2}=0.94, P>0.05\right)$. Urethral ultrasonography is a reliable and minimally invasive diagnostic tool for dysuria following TUPKP and is conducive to early treatment of dysuria following prostatectomy.
\end{abstract}

Keywords: Benign prostate hyperplasia (BPH), bipolar transurethral plasma kinetic prostatectomy (TUPKP), dysuria, ultrasonography

\section{Introduction}

Benign prostate hyperplasia (BPH) is a common disease in Department of Urinary Surgery where various minimally invasive therapies are extensively used. However, a number of patients still develop dysuria after such minimally invasive therapies, which was previously diagnosed mainly by urethrography and urinary cystoscopy. $806 \mathrm{BPH}$ patients who underwent bipolar transurethral plasma kinetic prostatectomy (TUPKP) between July 2006 and January 2011 in our center were retrospectively reviewed, and 120 patients complicating with postoperative dysuria were enrolled in this study. Ultrasonography was used for the diagnosis of postoperative dysuria. The report is presented as follows.

\footnotetext{
${ }^{1}$ These authors contributed equally to this study.

${ }^{*}$ Corresponding author: Bo Peng, Department of Urology, Shanghai Tenth People's Hospital, Tongji University School of Medicine, Shanghai, China. E-mail: pengbo6908@163.com.
} 
Table 1

The accordance and discrepancy rates of ultrasonography and urethral cystoscopy

\begin{tabular}{lccc}
\hline Diagnostic tool & Cases & Diagnose accordance rate & Diagnose discrepancy rate \\
\hline Urethral cystoscopy & 120 & $96.67 \%$ & $3.33 \%$ \\
Ultrasonography & 120 & $93.33 \%$ & $6.67 \%$ \\
\hline
\end{tabular}

\section{Patients and methods}

\subsection{Patients}

A total of 120 male patients developed dysuria (for $1 \sim 6$ months) after TUPKP ( 3 months $\sim 3$ years ago). Among them, 42 cases were complicated with urinary tract infection, 22 with diabetes, 10 with coronary artery diseases and 15 with hypertension. The mean age was 72.8 years (61 to 87 years). Before TUPKP, all patients were evaluated with digital rectal examination (DRE), transrectal ultrasonography (TRUS). Besides, the International Prostate Symptom Score (IPSS), quality of life score (QoLs), urodynamics assessment as well was prostate-specific antigen (PSA) were also evaluated. This study was approved by local Ethical Committee. All work was conducted in accordance with the Declaration of Helsinki (1964), and informed consents were obtained from all subjects.

\subsection{Ultrasonography}

The 120 patients received urethral ultrasonography (GE730, GE Company, USA) at a frequency of 6-12 MHz. A transrectal probe was used for examination of posterior urethra and a superficial one was used for examination of anterior urethra. When bladder was full of urine, external orifice of urethra was clamped with a penile clamp. Patients received urethral ultrasonography in a lateral position. The patients were asked to keep breath-holding urination state while the posterior urethra was observed with the anterior one in a supine position. Horizontal and longitudinal multi-section scans were performed. A normal human urethra is soft and elastic and internal urethral orifice is open during voiding. A representative sonogram manifestation was that the expanded urethra was substituted by liquid lucent space and the liquid flew into the distal urethra; then the profile was clear and the boundaries were completely smooth. The ultrasonography and urethroscopy results as well as findings during the surgery were analyzed and compared.

\section{Results}

Of the 120 patients, 22 presented with bladder neck closure, 20 with bladder orifice stricture (Fig. 1), 32 with membranous urethra stricture, 24 with pars cavernosa urethra stricture, 10 with prostate remnant, 2 with calculi in prostatic urethra and 2 with internal urethral orifice flap. The membranous urethra stricture had a length of $1.5 \sim 10 \mathrm{~mm}$ and an inner diameter of $1.5 \sim 4.2 \mathrm{~mm}$ in the narrow segment, while the pars cavernosa urethra stricture had a length of $5.1 \sim 16.0 \mathrm{~mm}$ and an inner diameter of $2.1 \sim 3.2 \mathrm{~mm}$ in the narrow segment. Results of urethral ultrasonography in these patients were completely consistent with the findings of urethral cystoscopy. No abnormal findings were detected in urethral ultrasonography of 8 patients. Urethral cystoscopy revealed that 4 patients had stenosis at external orifice of urethra and the other 4 patients had detrusor dysfunction with urodynamics study. Ultrasonography and urethral cystoscopy were used in combination in diagnosis of dysuria following TRPKP (Table 1). 

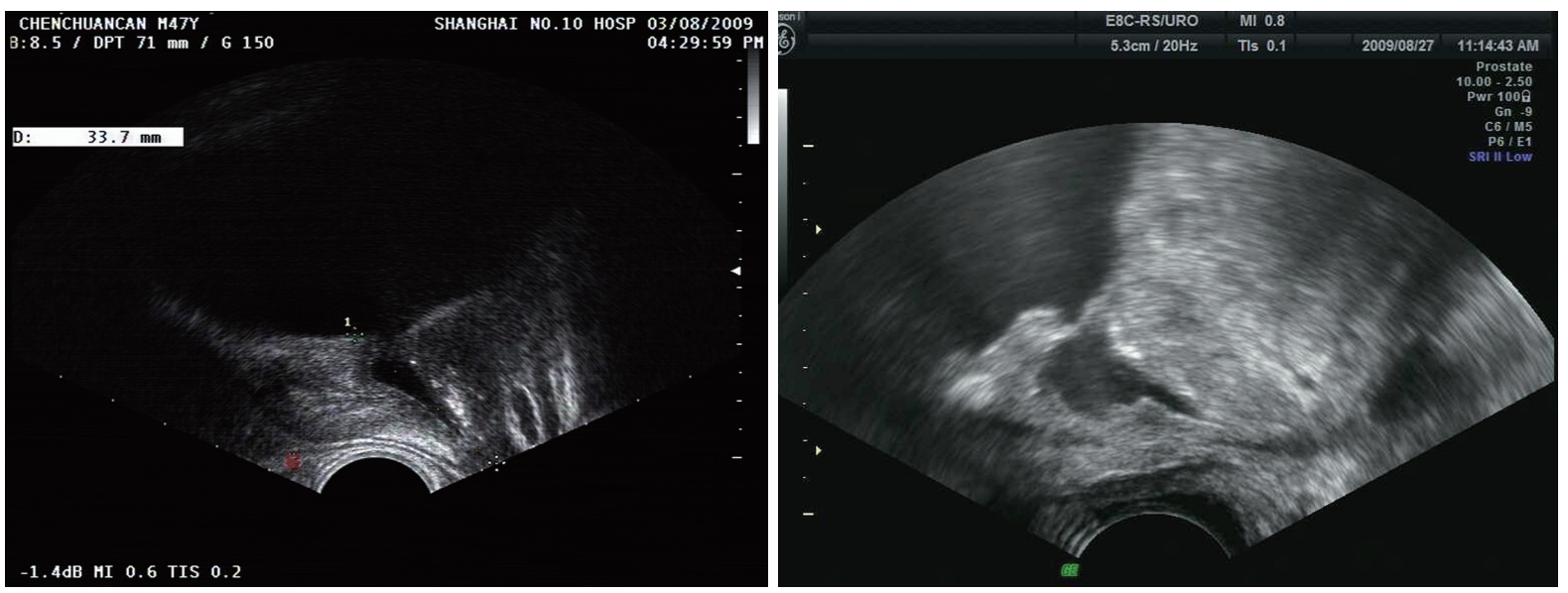

Fig. 1. Neck stricture and protruding of the hyperechoic zone toward the cavity were shown with closure of the internal urethral orifice during voiding.

The results of ultrasonography in 112 cases showed an effective rate of $93.33 \%$ in diagnosis of postoperative dysuria, while the results of urethral cystoscopy in 116 cases had an effective rate of $96.67 \%$. All data were analyzed by using SPSS 12.0 software (SPSS Inc, Chicago, IL). $\chi^{2}$-test was used for comparison of ultrasonography and urethral cystoscopy in the diagnosis of dysuria following TRPKP, while no significant difference was demonstrated between two diagnostic tools $\left(\chi^{2}=0.94, P>0.05\right)$.

\section{Discussion}

Bipolar transurethral plasma kinetic prostatectomy (TUPKP) has achieved an increased popularity worldwide currently due to the decreased incidence of electrotomy syndrome and increased safety with intraoperative saline irrigation. Despite the success in safety, some complications can still occur with TUPKP. The incidence of urethral stricture was $1.8 \% \sim 8.8 \%[1,2]$, which had no significant difference compared with common electrotomy or electro vaporization resection. Although urethrography is commonly used in the diagnosis of urethral stricture, a better tool is needed due to its inaccurate measurements in the length of narrow segment and X-ray radiation to physicians and patients during examination. Song, et al. reported that magnetic resonance imaging (MRI) could accurately localize urethral stricture and surrounding urethral scars, thus providing useful information in certain clinical situations, particularly posterior urethral trauma, and in the evaluation of the periurethral soft tissues [3]. However, MRI is relatively expensive. El-Ghar et al. reported that the role of MRI in analyzing the length of urethral stricture and the extent of scar was equivalent to urethrography and urethral ultrasonography, whereas MRI had advantages in detecting urethral stricture related soft tissue pathology [4]. Additionally, the threedimensional reconstruction was more commonly used in medical diagnosis. El-Kassaby and Sa reported three-dimensional spiral computed tomography/cysto-urethrography (CTCUG) and reconstruction pictures could provide physician preoperative evidence for better surgical planning and consequently better results of posterior urethral stricture [5,6]. Ultrasonography has got increased attention in the diagnosis of urethral stricture due to its characters such as presenting direct and real-time images as well as accurate information of exterior and interior structures of urethral lumen during urethral operations with good repeatability and without X-ray radiation [7]. 
The prostate, the cavernous body of the urethra and the lower border of the pubic symphysis, can be clearly shown in ultrasonography. The normal structure around the membranous urethra is hypoechoic. Its distal end is engorged bulbar urethra, which is smooth and beak-shaped, whose anterior aspect is just at the level of the lower border of pubic symphysis. With the help of apex of prostate, the membranous urethra can be well displayed by ultrasonography, as well as changes of structures of engorged urethral lumens and surrounding structures. Therefore, it is easy to locate and measure the length of urethral strictures and area of urethral stricture related peripheral scar using ultrasonography [8]. The urethral stricture in sonogram is presented as anechoic limitation in urethral cavity expansion, echo enhancement and thickening in the cavernous body and a strong echo accompanied by acoustic shadow in serious scar tissues. Moreover, the other urethral stricture related lesions could also be displayed, such as the secondary calculus, periurethral abscesses, diverticulum and false passages. Dynamic observation of fluid through the narrowed part of the urethral together with computer synchronizing acquisition and storage are helpful in the real-time monitoring of the narrowed segment. Urethrography alone can not measure the depth and extent of the scar possibly due to the overlap of contrast agent and inappropriate angels of photographs, which may, therefore, underestimate the length of urethral stricture. However, ultrasonography showed superiority in detecting scars around the narrowed segment and measuring the extent, which further determines the degree of spongiosum fibrosis and classification of urethral stricture. The degree of spongiosum fibrosis has critical effects on future therapies, preoperative planning and prognosis of urethral stricture. The ultrasonography is not only used in the repetitive real-time examination for the suspected lesions, but also in intraoperative monitoring to improve the accuracy and safety of the surgical procedures.

Among causes of secondary dysuria after TUPKP, such as urethral stricture, bladder neck closure, prostate remnant, calculi in the prostatic urethra, dysfunction of detrusor muscle of bladder and flap of internal urethral orifice, urethral stricture is the most common [9]. Common sites of postoperative urethral stricture are external orifice, suspension part, membranous part, prostate part of urethra and internal orifice. After clinical data in previous studies and this study was analyzed, the risk factors of postoperative urethral stricture were summarized as follows: 1) patients' physical conditions, such as scar formation, poor nutritional status or diabetic [10]; 2) injuries after excessive traction of the catheter or banding of the catheter by using absorbent gauze that compresses the penis, as well as a too thick catheter compressing the urethral mucosa and causing ischemia [11];3) inappropriate techniques such as forced insertion of the resectoscope into the urethra cause injury in the external orifice and the mucosa, resulting in postoperative urethral scar and stricture; 4) a large-sized prostate or unskilled technique during the operation, leads to an excessive operation time or repeated operation causing urethral injuries; 5) a too deep resection in neck of bladder or a too large power in vaporized resection leads to postoperative fibrosis; 6) the residual disinfectant damages the urethral mucosa resulting in postoperative strictures; not well controlled preoperative inflammation in the urinary system and improper postoperative care. Urethral stenosis usually presents with dysuria one month after electrotomy. The urethral ultrasonography manifestations of urethral stricture were as follows: 1) elevated echo of the urethral mucosa, a rough and narrow lumen, as well as limited opening of the narrow part during voiding; 2) scar tissues present as isoechoic, hyperechoic or heterogeneous echogenic mass protruding into the cavity: scar located in the bulbar urethra can lead to a better expansion of the urethral wall during voiding thus having little effect on urination; while scar in the membranous urethra can cause significant narrowing of the lumen so that urethra expansion is limited during urination; 3 ) different degrees of expansion proximal to the stenotic part; 4) absence of a normal funnel-shaped prostate fossa and a rough cavity rather than a smooth one, with hyperechoic or echogenic mass protruding into the cavity in the narrow part and failure to open the internal urethral orifice during urination in ultrasound of the prostate fossa [12]. 
Calculus of urethra in prostatic part was commonly formed in necrotic glandular tissues which usually presents as postoperative dysuria as well as further emiction interruption, hematuria and odynuria. Urethral ultrasound may reveal hyperechoic calculus in prostatic urethra accompanied by acoustic shadow. One case in this study had prostatic stones which were not detected by urethrography. Residual prostatic glandular tissues are more commonly seen in relatively bigger prostate with an inadequate resection of apex, leaving residual glandular tissues on both sides to constitute a closed phenomenon. It often presents as dysuria after TUPKP. Urethral ultrasonography may demonstrate isoechoic prostatic tissue nodules are protruding into the cavity of the prostatic urethra, which partially leads to urethral stricture during voiding. The flap of internal urethral orifice was rarely seen, which is more commonly observed in patients with prostate protruding towards the bladder and dysuria with emiction interruption is often seen postoperatively. Urethral ultrasonography may show an echogenic band in internal urethral orifice with one end attached to the urethral wall and the other end free with some activities during voiding. And the urination will be obstructed when the free end points to the urethral lumen. Of 120 cases in this study, two patients with flap of the internal urethral orifice were diagnosed by ultrasonography while not detected by contrast. Detrusor dysfunction usually presents as dysuria. Urinary ultrasonography may indicate closed urethra during resting and successful posterior urethral opening during voiding without stricture. Likewise, four patients were diagnosed with detrusor muscle dysfunction by urodynamics study.

Urethral ultrasonography is non-invasive and able to indicate the urethral lumen clearly, as well as the surrounding tissues of the posterior urethra, without contrast agent or X-ray irradiation. It is an ideal diagnostic tool in determining the localization and characters of the lesion and is useful in intraoperative monitoring for urethral stricture. However, urethral ultrasonography is not satisfactory for the display of the external urethral orifice, which is usually a time-consuming and complicated procedure, while urethrography is more reliable in detecting the urethral axial ray, false passage and pelvic deformity. Therefore, urethroscopy and urethrography should be used to examine such lesions and deformities.

\section{Acknowledgments}

This paper is supported by the National Basic Research Program of China (973 Program) under Grant No. 2011CB707700, the Knowledge Innovation Project of the Chinese Academy of Sciences under Grant No. KGCX2YW907, the National Natural Science Foundation of China under Grant No. 81027002, 81071205 and 30970778, the Science and Technology Key Project of Beijing Municipal Education Commission under Grant No. KZ200910005005, the Fellowship for Young International Scientists of the Chinese Academy of Sciences under Grant No. 2010Y2GA03.

\section{Conflict of interest}

The authors declare that they have no conflict of interest with any third party.

\section{References}

[1] R. Autorino, R. Damiano, G. Di Lorenzo, G. Quarto, S. Perdonà, M. D’Armiento and M. De Sio, Four-year outcome of a prospective randomised trial comparing bipolar plasmakinetic and monopolar transurethral resection of the prostate. Eur. Urol. 55, 922 (2009). 
[2] O. Sinanoglu, S. Ekici, M.N. Tatar, G. Turan, A. Keles and Z. Erdem, Postoperative outcomes of plasmakinetic transurethral resection of the prostate compared to monopolar transurethral resection of the prostate in patients with comorbidities. Urology. 80, 402 (2012).

[3] L. Song, M. Xie, Y. Zhang and Y. Xu, Imaging techniques for the diagnosis of male traumatic urethral strictures. J. Xray Sci. Technol. 21, 111 (2013).

[4] M.A. El-Ghar, Y. Osman, E. Elbaz, H. Refiae and T. El-Diasty, MR urethrogram versus combined retrograde urethrogram and sonourethrography in diagnosis of urethral stricture. Eur. J. Radiol. 74, 193 (2010).

[5] A.W. El-Kassaby, T. Osman, A. Abdel-Aal, M. Sadek and N. Nayef, Dynamic three-dimensional spiral computed tomographic cysto-urethrography: a novel technique for evaluating post-traumatic posterior urethral defects. B. J. U. Int. 92, 993 (2003).

[6] Y.L. Sa, Y.M. Xu, C. Feng, X.X. Ye and L.J. Song, Three-dimensional spiral computed tomographic cysto-urethrography for post-traumatic complex posterior urethral strictures associated with urethral-rectal fistula. J. Xray Sci. Technol. 21, 133 (2013).

[7] J. Seibold, M. Werther, S. Alloussi, T. Todenhöfer, G. Gakis, S. Aufderklamm, D. Schilling, A. Stenzl and C. Schwentner, Urethral ultrasound as a screening tool for stricture recurrence after oral mucosa graft urethroplasty. Urology. 78, 696 (2011).

[8] E.M. Gong, C.M. Arellano, J.S. Chow and R.S. Lee, Sonourethrogram to manage adolescent anterior urethral stricture. J. Urol. 184, 1699 (2010).

[9] D.P. Michielsen and D. Coomans, Urethral strictures and bipolar transurethral resection in saline of the prostate: fact or fiction? J. Endourol. 24, 1333 (2010).

[10] G.H. Jordan, E.A. Eltahawy and O.R. Virasor, The technique of vessel sparing excision and primary anastomosis for proximal bulbous urethral reconstruction. J. Urol. 177, 1799 (2007).

[11] J. Xu, H.N. Li and L.M. Qu, Causes and Prevention of Urethral Stricture after Transurethral Resection of the Prostate. Chin. J. Min. Inv. Surg. (in Chinese). 9, 976 (2009).

[12] S. Mandal and A. Goel, Impact of urethral ultrasonography on decision-making in anterior urethroplasty. B. J. U. Int. 109,35 (2012). 